\title{
Qualidade de maçãs 'Fuji' armazenadas em atmosfera controlada e influência do clima na degenerescência da polpa
}

\author{
Thais Roseli Corrêa ${ }^{(1)}$, Cristiano André Steffens ${ }^{(1)}$, Cassandro Vidal Talamini do Amarante ${ }^{(1)}$, Auri Brackmann $^{(2)}$, \\ João Paulo Generoso Silveira ${ }^{(1)}$, Hélio Tanaka ${ }^{(1)}$ e Vanderlei Both ${ }^{(2)}$
}

(1)Universidade do Estado de Santa Catarina, Centro de Ciências Agroveterinárias, Programa de Pós-Graduação em Ciências Agrárias, Avenida Luiz de Camões, nํ2 2.090, CEP 88520-000 Lages, SC. E-mail: thaisroselicorrea@hotmail.com, steffens@cav.udesc.br, amarante@cav.udesc.br, joaop-silveira@hotmail.com, tanaka.helio@hotmail.com (2)Universidade Federal de Santa Maria, Centro de Ciências Rurais, Departamento de Fitotecnia, CEP 99105-900 Santa Maria, RS. E-mail: brackman@ccr.ufsm.br, vanderleiboth@yahoo.com.br

\begin{abstract}
Resumo - O objetivo deste trabalho foi avaliar a qualidade de frutos e a relação entre condições climáticas durante o desenvolvimento dos frutos e incidência de degenerescência da polpa no armazenamento em atmosfera controlada (AC) de maçãs 'Fuji'. Foram utilizados frutos de três pomares (Vacaria, RS, e São Joaquim e Lages, SC) e de dois anos agrícolas (2006/2007 e 2007/2008). Durante o desenvolvimento dos frutos, os pomares foram monitorados diariamente, quanto a temperaturas mínimas, médias e máximas, umidade relativa e precipitação. Os frutos foram armazenados por oito meses em diferentes condições de $\mathrm{AC}, \mathrm{a}-0,5 \pm 0,1^{\circ} \mathrm{C}$ e umidade relativa de $97 \%$. Foram estimadas as correlações de Pearson entre as variáveis relativas às condições climáticas e a incidência de degenerescência da polpa após o armazenamento sob AC. Frutos armazenados sob 1,2 $\mathrm{kPa}$ de $\mathrm{O}_{2}$ e 2,0 $\mathrm{kPa}$ de $\mathrm{CO}_{2}$ apresentaram maior acidez titulável, firmeza de polpa e textura e menores taxas respiratória e de produção de etileno; exibiram, porém, alta incidência de degenerescência da polpa, em comparação aos armazenados sob 1,2 $\mathrm{kPa}$ de $\mathrm{O}_{2} \mathrm{e}<0,5 \mathrm{kPa}$ de $\mathrm{CO}_{2}$. A incidência de degenerescência da polpa diminui com o aumento nas temperaturas médias diárias, ocorridas entre 90 e 210 dias após o pleno florescimento.
\end{abstract}

Termos para indexação: Malus domestica, armazenamento, distúrbios fisiológicos, pingo-de-mel, pós-colheita, qualidade de frutos.

\section{Quality of 'Fuji' apples stored under controlled atmosphere and influence of climate on the incidence of internal browning}

\begin{abstract}
The objective of this work was to evaluate fruit quality and the relation between climatic conditions during fruit development and internal browning incidence under controlled atmosphere (CA) of storage, in 'Fuji' apples. Fruit from three orchards (Vacaria, RS, and São Joaquim and Lages, SC) and two years (2006/2007 e 2007/2008) were evaluated. The orchards were daily monitored, during fruit development, for minimum, medium and maximum temperatures, and for relative humidity and rainfall. Fruit were stored during eight months in different CA conditions, at $-0.5 \pm 0,1^{\circ} \mathrm{C}$ and $97 \%$ relative humidity. Pearson's correlations between climatic condition variables and internal browning incidence after $\mathrm{CA}$ storage were estimated. Fruit stored under $1.2 \mathrm{kPa}$ of $\mathrm{O}_{2}$ and $2.0 \mathrm{kPa}$ of $\mathrm{CO}_{2}$ had higher titratable acidity, flesh firmness and texture, and lower respiration and ethylene production rates; however these fruit exhibited a high internal browning incidence in comparison to fruit stored at $1.2 \mathrm{kPa}$ of $\mathrm{O}_{2}$ and $<0.5 \mathrm{kPa}$ of $\mathrm{CO}_{2}$. The internal browning incidence decreases as the medium daily temperatures occurred between 90 and 210 days after full bloom increase.
\end{abstract}

Index terms: Malus domestica, storage, physiological disorders, water core, postharvest, fruit quality.

\section{Introdução}

A condição ideal de armazenamento em atmosfera controlada (AC), para maçãs 'Fuji', envolve pressões parciais de $\mathrm{O}_{2}$ de 1,0 a $1,2 \mathrm{kPa}$, com $\mathrm{CO}_{2}$ abaixo de 0,5 $\mathrm{kPa}$ (Brackmann et al., 2002a). No entanto, o armazenamento prolongado (oito meses) dos frutos nessas condições pode causar acentuada redução da acidez e amarelecimento dos frutos, além de perdas quantitativas, em consequência da elevada incidência de podridões. A atmosfera controlada com 2,0 kPa de $\mathrm{CO}_{2}$ pode proporcionar maior firmeza de polpa e acidez titulável, cor da epiderme mais verde e menor ocorrência de podridões (Brackmann et al., 2002a), porém com maior risco de ocorrer o desenvolvimento de degenerescência da polpa. 
A degenerescência de polpa em maçãs 'Fuji' é um distúrbio caracterizado pelo escurecimento na região do córtex, que não atinge a região carpelar (Clarck et al., 2003; Brackmann et al., 2005b), e está associada a altas pressões parciais de $\mathrm{CO}_{2}$ ou a baixas pressões parciais de $\mathrm{O}_{2}$ (Brackmann et al., 2002a; Pedreschi et al., 2008). A ocorrência de degenerescência da polpa, associada à sensibilidade dos frutos ao $\mathrm{CO}_{2}$, pode também estar relacionada à incidência de "pingo-de-mel" no momento da colheita. O pingo-de-mel é um distúrbio pré-colheita muito frequente na maçã 'Fuji' e caracteriza-se por apresentar áreas de aparência translúcida na polpa, em razão da inundação dos espaços intercelulares com suco rico em sorbitol (Bowen \& Watkins, 1997). Quanto maior for a severidade desse distúrbio, maior será a sensibilidade dos frutos aos danos causados por $\mathrm{CO}_{2}$ (Brackmann et al., 2002a). Portanto, a incidência e severidade de degenerescência da polpa podem variar conforme o ano e o local de produção (Brackmann et al., 2002b).

A temperatura durante o crescimento e maturação dos frutos, o tipo de solo, a nutrição mineral, a idade da planta e o manejo do pomar podem exercer forte influência sobre o desenvolvimento de degenerescência da polpa (Ferguson et al., 1999). Lau (1997) menciona que a incidência de degenerescência da polpa, em frutos armazenados em $\mathrm{AC}$, pode estar relacionada à baixa difusividade do $\mathrm{O}_{2}$ e $\mathrm{CO}_{2}$ no tecido da polpa, em conseqüência do adensamento das células, o que prejudicaria as trocas gasosas. Esse autor considera que a temperatura do ar durante o desenvolvimento dos frutos pode influenciar o adensamento das células, e que a degenerescência da polpa prevalece em frutos de plantas que se desenvolvem em regiões frias. Segundo Ferguson et al. (1999), além da temperatura durante o crescimento e maturação dos frutos, a radiação solar, a umidade relativa e a precipitação pluviométrica também podem causar maior ocorrência de distúrbios fisiológicos. Portanto, o conhecimento dos fatores climáticos que influenciam a sensibilidade dos frutos aos danos causados pelo $\mathrm{CO}_{2}$, durante o armazenamento em condições de AC, pode permitir a identificação de locais de produção e de anos agrícolas em que as condições climáticas favoreçam o desenvolvimento de degenerescência da polpa.

O objetivo deste trabalho foi avaliar a qualidade de frutos e a relação entre condições climáticas durante o desenvolvimento dos frutos e incidência de degenerescência da polpa no armazenamento em atmosfera controlada (AC) de maçãs 'Fuji'.

\section{Material e Métodos}

Dois experimentos foram conduzidos com maçãs da cultivar Fuji: um com frutos provenientes de pomares comerciais dos municípios de Lages, SC, São Joaquim, SC, e Vacaria, RS, no ano agrícola 2006/2007, e outro com frutos de pomares de Lages e São Joaquim, em 2007/2008. Em ambos os anos, os frutos foram colhidos 210 dias após o pleno florescimento.

Após a colheita, frutos livres de incidência de podridões, lesões, defeitos ou ferimentos foram transportados para o Laboratório do Núcleo de Pesquisa e Pós-Colheita, da Universidade Federal de Santa Maria, em Santa Maria, RS.

Os tratamentos avaliados foram compostos pela combinação das condições climáticas de três regiões de cultivo com duas condições de atmosfera controlada: $1,2 \mathrm{kPa}$ de $\mathrm{O}_{2}+<0,5 \mathrm{kPa}$ de $\mathrm{CO}_{2}$ e $1,2 \mathrm{kPa}$ de $\mathrm{O}_{2}+$ $2,0 \mathrm{kPa}$ de $\mathrm{CO}_{2}$, a $-0,5 \pm 0,1^{\circ} \mathrm{C}$ e umidade relativa de $97 \%$. O delineamento experimental utilizado foi o inteiramente casualizado, em esquema fatorial completo 2x3, em 2006/2007, e 2x2, em 2007/2008. Cada unidade experimental foi constituída por 25 frutos.

Os frutos de todos os tratamentos foram armazenados em minicâmaras experimentais com capacidade de $250 \mathrm{~L}$. As pressões parciais de $\mathrm{O}_{2}$ foram obtidas mediante a diluição do $\mathrm{O}_{2}$ no ambiente de armazenamento, com injeção de $\mathrm{N}_{2}$ proveniente de um gerador de nitrogênio (Janus \& Pergher, Porto Alegre, Brasil), que utiliza o princípio "pressure swing adsorption" (PSA). A pressão parcial de $\mathrm{CO}_{2}$, referente ao tratamento com alto $\mathrm{CO}_{2}$ $(2,0 \mathrm{kPa})$, foi obtida mediante a injeção desse gás, proveniente de cilindro de alta pressão. A manutenção das pressões parciais desejáveis dos gases, nas duas condições de armazenamento, que variavam em razão da respiração dos frutos, foi realizada, diariamente, com o uso de equipamento automático para controle de gases (Kronenberger/Climasul, Caxias do Sul, RS, Brasil). Quando os níveis do $\mathrm{CO}_{2}$ e $\mathrm{O}_{2}$ não estavam adequados, o equipamento procedia à correção das pressões parciais até os níveis preestabelecidos nos tratamentos. O oxigênio consumido pela respiração foi reposto por meio da injeção de ar atmosférico nas minicâmaras, e o $\mathrm{CO}_{2} \mathrm{em}$ excesso (no tratamento $\operatorname{com} 2,0 \mathrm{kPa}$ ) foi absorvido por uma solução de hidróxido 
de potássio a $40 \%$, através da qual foi circulado o ar do ambiente de armazenamento. No tratamento com baixo $\mathrm{CO}_{2}$, a pressão parcial $<0,5 \mathrm{kPa}$ foi mantida por meio da colocação de cal hidratada no interior das minicâmaras, para a contínua eliminação do $\mathrm{CO}_{2}$ no ambiente de armazenamento.

Os frutos foram analisados após oito meses de armazenamento e após sete dias de exposição à temperatura ambiente $\left(20^{\circ} \mathrm{C}\right)$, para simular o período de comercialização. As características analisadas foram: taxa respiratória e de produção de etileno, acidez titulável (AT), teores de sólidos solúveis (SS), firmeza de polpa, cor da epiderme, incidências de podridões, podridão carpelar e degenerescência da polpa.

A taxa respiratória (nmol de $\mathrm{CO}_{2} \mathrm{~kg}^{-1} \mathrm{~s}^{-1}$ ) e de produção de etileno ( $\mathrm{pmol}$ de $\mathrm{C}_{2} \mathrm{H}_{4} \mathrm{~kg}^{-1} \mathrm{~s}^{-1}$ ) foram quantificadas por cromatografia gasosa (Brackmann et al., 2002a). Quinze frutos de cada amostra foram colocados em recipiente de plástico, com o volume de $4.100 \mathrm{~mL}$ e fechamento hermético. A taxa respiratória foi obtida pela diferença da concentração de $\mathrm{CO}_{2}$ no interior do recipiente imediatamente após o seu fechamento e depois de uma hora. Alíquotas de $1 \mathrm{~mL}$ de gás foram retiradas dos recipientes com uso de um septo, e injetadas em um cromatógrafo à gás, modelo CP-3800 (Varian, Palo Alto, EUA), equipado com uma coluna Porapak N de $3 \mathrm{~m}$ de comprimento (80-100 mesh), metanador e detector de ionização de chama. As temperaturas da coluna, do detector, do metanador e do injetor foram de $45,120,300$ e $110^{\circ} \mathrm{C}$, respectivamente. Os fluxos de nitrogênio, hidrogênio e ar sintético foram de 70, 30 e $300 \mathrm{~mL} \mathrm{~min}^{-1}$, respectivamente.

O teste de iodo-amido foi determinado por meio da comparação do escurecimento da metade peduncular dos frutos tratada com uma solução de iodo, com a da tabela de fotografias desenvolvidas por Streif (1984), em que o índice 1 indica o teor máximo de amido e o índice 5 representa o amido totalmente hidrolisado.

A densidade dos frutos, expressa em gramas por mililitros, foi estimada pela relação entre a massa e o volume, por meio do deslocamento da coluna de água, após a imersão completa do fruto em recipiente com $800 \mathrm{~mL}$ de água.

O diâmetro dos frutos $(\mathrm{mm})$ foi calculado em duas medições com paquímetro, na secção transversal de cada fruto, com posterior cálculo do diâmetro médio.
O índice de cor vermelha (ICV) foi determinado pela avaliação da superfície dos frutos recoberta com coloração vermelha, à qual foram atribuídas notas de 1 a 4 para as percentagens da superfície dos fruto pigmentada de vermelho de $0-25,26-50,51-75$ e 76-100\%, respectivamente (Steffens et al., 2006).

A determinação da cor da epiderme (ângulo hue) foi efetuada com um colorímetro Minolta, modelo CR 400 (Minolta, Osaka, Japão). As determinações foram realizadas na região equatorial dos frutos, e os resultados foram expressos em ângulo de cor $\left(\mathrm{h}^{\circ}\right)$, que define a coloração básica, em que $0^{\circ}$ é a cor vermelha, $90^{\circ}$ é a cor amarela e $180^{\circ}$ é a verde.

A firmeza de polpa $(\mathrm{N})$ foi determinada na região equatorial dos frutos, em dois lados diametralmente opostos, com o auxílio de um penetrômetro equipado com ponteira de $11 \mathrm{~mm}$ de diâmetro, após remoção de uma pequena porção da epiderme (Brackmann et al., 2002a).

Os valores da AT (meq $100 \mathrm{~mL}^{-1}$ ) foram obtidos por meio de uma amostra de $10 \mathrm{~mL}$ de suco, extraído de fatias transversais retiradas da região equatorial dos frutos, em uma centrífuga. Esta amostra foi diluída em $90 \mathrm{~mL}$ de água destilada e titulada com solução de $\mathrm{NaOH} 0,1 \mathrm{~N}$, até $\mathrm{pH} 8,1$, o qual foi determinado com o auxílio de um peagâmetro digital (Oakto, Singapura, Singapura), (Brackmann et al., 2002a). Os teores de SS foram determinados por refratometria ( $\left.{ }^{\circ} \mathrm{Brix}\right)$, com correção do efeito da temperatura (Brackmann et al., 2002a)

A incidência e a severidade de pingo-de-mel foram determinadas conforme metodologia descrita por Bowen \& Watkins (1997). A incidência de podridões foi avaliada pela percentagem de frutos afetados, com lesões externas maiores do que $5 \mathrm{~mm}$ de diâmetro, com características de infecção de patógenos, tecido mole, aquoso e de coloração bege, tanto na epiderme quanto na polpa, com ou sem massas brancas e azuis constituídas de micélio e esporos de fungos (Sanhueza \& Betti, 2005). A podridão carpelar foi avaliada pela percentagem de frutos que apresentavam sintoma interno característico de infecção por fungos, nas sementes e na cavidade carpelar (carpelos), conforme Kretzschmar et al. (2007).

A percentagem de incidência de degenerescência da polpa foi avaliada por meio de um corte na secção transversal dos frutos e de contagem dos frutos que apresentavam qualquer tipo de escurecimento, aspecto 
úmido ou seco e esponjoso, e pequenas cavidades, nas regiões internas da polpa (Brackmann et al., 1999).

Durante a fase de desenvolvimento dos frutos, foram coletados os dados diários de precipitação pluviométrica, umidade relativa e temperaturas mínimas, médias e máximas, registrados em estações metereológicas instaladas nos pomares.

Os dados obtidos aos oito meses de armazenamento e após sete dias em condição ambiente, foram submetidos à análise da variância (ANOVA). Dados em percentagem foram transformados pela fórmula arcoseno $[(\mathrm{x}+0,5) / 100]^{0,5}$, antes de serem submetidos à ANOVA. Para a comparação das médias, adotou-se o teste de Tukey a $5 \%$ de probabilidade. As médias das variáveis analisadas na colheita, após o armazenamento, e os dados climáticos coletados durante o ciclo de desenvolvimento dos frutos foram analisados conjuntamente para as duas safras, e submetidos ao teste de correlação de Pearson com a incidência da degenerescência da polpa.

\section{Resultados e Discussão}

As condições climáticas nos pomares de Vacaria, São Joaquim e Lages, nos dois anos agrícolas, estão sumarizadas na Tabela 1. Em geral, não houve efeito dos pomares de origem dos frutos nas variáveis analisadas.

No momento da saída da câmara e após sete dias de exposição dos frutos à condição ambiente, no ano agrícola 2007/2008, a taxa respiratória não apresentou diferenças entre as condições de armazenamento (Tabela 2). Porém, no ano agrícola 2006/2007, os frutos armazenados com 2,0 $\mathrm{kPa}$ de $\mathrm{CO}_{2}$ apresentaram menor

Tabela 1. Dados climáticos de temperatura e precipitação pluviométrica de pomares, dos anos agrícolas de 2006/2007 e 2007/2008.

\begin{tabular}{lccc}
\hline Dados climáticos & Vacaria & São Joaquim & Lages \\
\hline & \multicolumn{3}{c}{$2006 / 2007$} \\
Temperatura mínima $\left({ }^{\circ} \mathrm{C}\right)$ & 13,4 & 12,3 & 14,0 \\
Temperatura média $\left({ }^{\circ} \mathrm{C}\right)$ & 19,0 & 18,3 & 19,2 \\
Temperatura máxima $\left({ }^{\circ} \mathrm{C}\right)$ & 24,6 & 24,2 & 24,4 \\
Precipitação $(\mathrm{mm})$ & 315,3 & 133,5 & 136,0 \\
Umidade relativa mínima (\%) & 58,3 & 55,4 & 52,1 \\
Umidade relativa máxima (\%) & 92,9 & 95,8 & 90,3 \\
\hline & & $2007 / 2008$ & - \\
Temperatura mínima $\left({ }^{\circ} \mathrm{C}\right)$ & 11,0 & 11,4 & - \\
Temperatura média $\left({ }^{\circ} \mathrm{C}\right)$ & 18,9 & 17,8 & - \\
Temperatura máxima $\left({ }^{\circ} \mathrm{C}\right)$ & 26,8 & 24,2 & - \\
Precipitação (mm) & 113,4 & 155,6 & - \\
Umidade relativa mínima $(\%)$ & 55,1 & 58,2 & - \\
Umidade relativa máxima $(\%)$ & 95,3 & 94,9 &
\end{tabular}

taxa respiratória, após sete dias em condição ambiente, e não foram verificadas diferenças entre pomares para essa variável. Saquet \& Streif (2002) e Steffens et al. (2007) verificaram menor taxa respiratória de maçãs em condições com baixo $\mathrm{O}_{2}$ e alto $\mathrm{CO}_{2}$. $\mathrm{O}$ efeito da $\mathrm{AC}$ sobre a redução da respiração celular resulta da menor atividade das enzimas da glicólise e do ciclo de Krebs, em condições de baixas pressões parciais de $\mathrm{O}_{2}$ e altas pressões parciais de $\mathrm{CO}_{2}$ (Brackmann et al., 2005c).

Em ambos os anos agrícolas, a taxa de produção de etileno foi menor nos frutos armazenados na condição $1,2 \mathrm{kPa}$ de $\mathrm{O}_{2}+2,0 \mathrm{kPa}$ de $\mathrm{CO}_{2}$, tanto no momento da saída da câmara fria, quanto após sete dias de exposição dos frutos à condição ambiente (Tabela 2). Entre os pomares, em cada ano, não foram observadas diferenças significativas. A menor taxa de produção de etileno no tratamento com $2,0 \mathrm{kPa}$ de $\mathrm{CO}_{2}$ deve estar relacionada ao efeito inibitório do $\mathrm{CO}_{2}$ na ação do etileno, que teria reduzida a sua produção autocatílica (Blankenship \& Dole, 2003).

Após oito meses de armazenamento e após 7 dias de exposição à condição ambiente, em ambos os anos, os frutos armazenados na condição de $1,2 \mathrm{kPa} \mathrm{O}_{2}$ e 2,0 $\mathrm{kPa} \mathrm{CO}_{2}$ apresentaram maior firmeza de polpa do que os frutos armazenados na condição $1,2 \mathrm{kPa}$ de $\mathrm{O}_{2} \mathrm{e}$ $<0,5 \mathrm{kPa}$ de $\mathrm{CO}_{2}$ (Tabela 3). Em relação aos pomares, não

Tabela 2. Taxas respiratórias e de produção de etileno em maçãs 'Fuji', após oito meses de armazenamento em duas condições de atmosfera controlada, e mais sete dias de exposição dos frutos a $20^{\circ} \mathrm{C}$. As médias dos tratamentos referem-se a pomares de três municípios produtores, em 2006/2007, e de dois, em 2007/2008 ${ }^{(1)}$.

\begin{tabular}{|c|c|c|}
\hline Pressões parciais de $\mathrm{O}_{2}$ e $\mathrm{CO}_{2}$ & $2006 / 2007$ & $2007 / 2008$ \\
\hline & \multicolumn{2}{|c|}{ Taxa respiratória $\left(\mathrm{nmol} \mathrm{CO} \mathrm{kg}^{-1} \mathrm{~s}^{-1}\right)$} \\
\hline \multicolumn{3}{|l|}{ Saída da câmara } \\
\hline $1,2 \mathrm{kPa}$ de $\mathrm{O}_{2}+<0,5 \mathrm{kPa}$ de $\mathrm{CO}_{2}$ & $5,91 \mathrm{a}$ & $3,26 \mathrm{a}$ \\
\hline $1,2 \mathrm{kPa}$ de $\mathrm{O}_{2}+2,0 \mathrm{kPa}$ de $\mathrm{CO}_{2}$ & $7,57 \mathrm{a}$ & $3,96 a$ \\
\hline $\mathrm{CV}(\%)$ & 16,4 & 14,9 \\
\hline \multicolumn{3}{|l|}{ Após sete dias a $20^{\circ} \mathrm{C}$} \\
\hline $1,2 \mathrm{kPa}$ de $\mathrm{O}_{2}+<0,5 \mathrm{kPa}$ de $\mathrm{CO}_{2}$ & $11,8 \mathrm{a}$ & $4,86 a$ \\
\hline $1,2 \mathrm{kPa}$ de $\mathrm{O}_{2}+2,0 \mathrm{kPa}$ de $\mathrm{CO}_{2}$ & $9,6 \mathrm{~b}$ & $5,02 \mathrm{a}$ \\
\hline \multirow[t]{2}{*}{$\mathrm{CV}(\%)$} & 11,4 & 32,9 \\
\hline & \multicolumn{2}{|c|}{ Taxa de produção de etileno $\left(\mathrm{pmol} \mathrm{kg}^{-1} \mathrm{~s}^{-1}\right.$} \\
\hline \multicolumn{3}{|l|}{ Saída da câmara } \\
\hline $1,2 \mathrm{kPa}$ de $\mathrm{O}_{2}+<0,5 \mathrm{kPa}$ de $\mathrm{CO}_{2}$ & $1,18 \mathrm{a}$ & $0,42 \mathrm{a}$ \\
\hline $1,2 \mathrm{kPa}$ de $\mathrm{O}_{2}+2,0 \mathrm{kPa}$ de $\mathrm{CO}_{2}$ & $0,10 \mathrm{~b}$ & $0,12 b$ \\
\hline CV $(\%)$ & 19,3 & 20,4 \\
\hline \multicolumn{3}{|l|}{ Após sete dias a $20^{\circ} \mathrm{C}$} \\
\hline $1,2 \mathrm{kPa}$ de $\mathrm{O}_{2}+<0,5 \mathrm{kPa}$ de $\mathrm{CO}_{2}$ & $1,45 \mathrm{a}$ & $1,60 \mathrm{a}$ \\
\hline $1,2 \mathrm{kPa}$ de $\mathrm{O}_{2}+2,0 \mathrm{kPa}$ de $\mathrm{CO}_{2}$ & $0,54 \mathrm{~b}$ & $0,74 \mathrm{~b}$ \\
\hline $\mathrm{CV}(\%)$ & 26,2 & 37,9 \\
\hline
\end{tabular}


foram observadas diferenças significativas. $\mathrm{O}$ resultado obtido está de acordo com Brackmann et al. (2002a), que observaram maiores valores de firmeza de polpa em maçãs 'Fuji' armazenadas sob alta pressão de $\mathrm{CO}_{2}$. Konopacka \& Plocharski (2004) encontraram maiores valores de firmeza de polpa ao armazenar maçãs 'Elstar', 'Jonagold' e 'Gloster' em AC com maiores níveis de $\mathrm{CO}_{2}$. Brackmann et al. (2002b) afirmaram que a manutenção da firmeza de polpa, em maçãs armazenadas em AC, é consequência da menor atividade de enzimas hidrolíticas da parede celular resultante da combinação de baixa pressão de $\mathrm{O}_{2}$ e alta de $\mathrm{CO}_{2}$.

Os maiores valores de AT, após oito meses de armazenamento e mais sete dias de exposição dos frutos a $20^{\circ} \mathrm{C}$, foram obtidos na condição de $1,2 \mathrm{kPa} \mathrm{O}$ e 2,0 $\mathrm{kPa} \mathrm{CO}$ (Tabela 3), no ano agrícola 2006/2007. Contudo, entre pomares, não houve diferença significativa. Possivelmente, os maiores valores de AT estão relacionados ao menor consumo de ácidos durante o período de armazenamento, pois pressões parciais mais elevadas de $\mathrm{CO}_{2}$ reduzem a atividade respiratória dos frutos durante o armazenamento (Saquet \& Streif, 2002; Steffens et al., 2007). No ano agrícola 2007/2008, no entanto, não foram observadas diferenças significativas nos valores de AT entre as duas condições de armazenamento.

No primeiro ano avaliado, os teores de SS não diferiram entre as condições de armazenamento (Tabela 3) e, também, não houve diferença significativa entre os pomares. No segundo ano, os teores de SS foram maiores nos frutos armazenados sob alta pressão de $\mathrm{CO}_{2}$ do que nos frutos oriundos do pomar de São Joaquim. Brackmann et al. (2005b), não verificaram diferenças nos teores de SS de maçãs 'Fuji', em resposta a diferentes condições de AC. Esses autores consideram que as condições de AC, de modo geral, não causam efeito consistente sobre os teores de SS. Contudo, no presente trabalho, no segundo ano de realização do experimento, observou-se efeito das condições de AC em frutos do pomar de São Joaquim.

A cor de fundo da epiderme não apresentou diferença entre as condições de armazenamento, no primeiro ano avaliado (Tabela 3). Contudo, no segundo ano, os frutos armazenados na condição de $\mathrm{AC}$ com 2,0 kPa de $\mathrm{CO}_{2}$ apresentaram cor de fundo da epiderme mais verde $\left(\right.$ maior $\left.\mathrm{h}^{\circ}\right)($ Tabela2). Entre os pomares, não foi observada diferença significativa em ambos os anos. Brackmann et al. (2000), ao armazenar maçãs 'Gala' em diferentes condições de $\mathrm{AC}$, não encontraram diferenças quanto à cor de fundo da epiderme. Entretanto, Brackmann et al. (2005a), ao armazenar maçãs dessa mesma cultivar, observaram maior retenção da cor verde na epiderme com a elevação do $\mathrm{CO}_{2}$ durante o armazenamento em AC. Esse efeito de condições de AC, em determinados anos de avaliação na mesma cultivar, evidencia que fatores pré-colheita possivelmente exercem influência sobre a cor da epiderme.

Quanto à incidência de podridões, não houve diferenças significativas entre as condições de armazenamento (Tabela 4). Brackmann et al. (2002b)

Tabela 3. Firmeza de polpa, acidez titulável, sólidos solúveis e cor da epiderme em maçãs 'Fuji', após oito meses de armazenamento em duas condições de atmosfera controlada, e mais sete dias de exposição dos frutos a $20^{\circ} \mathrm{C}^{(1)}$.

\begin{tabular}{|c|c|c|c|c|c|c|c|}
\hline \multirow{2}{*}{ Pressões parciais de $\mathrm{O}_{2}$ e $\mathrm{CO}_{2}$} & \multicolumn{4}{|c|}{$2006 / 2007$} & \multicolumn{3}{|c|}{$2007 / 2008$} \\
\hline & Vacaria & São Joaquim & Lages & Média & Vacaria & São Joaquim & Média \\
\hline & \multicolumn{7}{|c|}{ Firmeza de polpa (N) } \\
\hline $1,2 \mathrm{kPa}$ de $\mathrm{O}_{2}+<0,5 \mathrm{kPa}$ de $\mathrm{CO}_{2}$ & $-(2)$ & - & - & $60,1 b$ & - & - & $62,4 b$ \\
\hline $1,2 \mathrm{kPa}$ de $\mathrm{O}_{2}+2,0 \mathrm{kPa}$ de $\mathrm{CO}_{2}$ & - & - & - & $62,8 \mathrm{a}$ & - & - & $69,6 \mathrm{a}$ \\
\hline \multirow[t]{2}{*}{ CV $(\%)$} & & 3,1 & & & & & \\
\hline & \multicolumn{7}{|c|}{ Acidez titulável (meq 100 mL $^{-1}$ ) } \\
\hline $1,2 \mathrm{kPa}$ de $\mathrm{O}_{2}+<0,5 \mathrm{kPa}$ de $\mathrm{CO}_{2}$ & - & - & - & $3,87 \mathrm{~b}$ & - & - & $4,86 \mathrm{a}$ \\
\hline $1,2 \mathrm{kPa}$ de $\mathrm{O}_{2}+2,0 \mathrm{kPa}$ de $\mathrm{CO}_{2}$ & - & - & - & $4,46 \mathrm{a}$ & - & - & $5,02 \mathrm{a}$ \\
\hline \multirow[t]{2}{*}{ CV $(\%)$} & & 7,5 & & & & & \\
\hline & \multicolumn{7}{|c|}{ Sólidos solúveis ( $\left.{ }^{\circ} \mathrm{Brix}\right)$} \\
\hline $1,2 \mathrm{kPa}$ de $\mathrm{O}_{2}+<0,5 \mathrm{kPa}$ de $\mathrm{CO}_{2}$ & - & - & - & $13,8 \mathrm{a}$ & $12,8 \mathrm{a}$ & $12,6 b$ & $12,7 b$ \\
\hline $1,2 \mathrm{kPa}$ de $\mathrm{O}_{2}+2,0 \mathrm{kPa}$ de $\mathrm{CO}_{2}$ & - & - & - & $13,8 \mathrm{a}$ & $12,8 \mathrm{a}$ & $14,4 \mathrm{a}$ & $13,6 \mathrm{a}$ \\
\hline \multirow[t]{2}{*}{ CV $(\%)$} & & 1,6 & & & & & \\
\hline & \multicolumn{7}{|c|}{ Cor da epiderme $\left(\mathrm{h}^{\circ}\right)$} \\
\hline $1,2 \mathrm{kPa}$ de $\mathrm{O}_{2}+<0,5 \mathrm{kPa}$ de $\mathrm{CO}_{2}$ & - & - & - & $91,1 \mathrm{a}$ & - & - & $90,7 b$ \\
\hline $1,2 \mathrm{kPa}$ de $\mathrm{O}_{2}+2,0 \mathrm{kPa}$ de $\mathrm{CO}_{2}$ & - & - & - & $92,1 \mathrm{a}$ & - & - & $95,3 \mathrm{a}$ \\
\hline $\mathrm{CV}(\%)$ & & 1,9 & & & & & \\
\hline
\end{tabular}


encontraram redução na incidência de podridões em maçãs 'Braeburn', com a elevação do $\mathrm{CO}_{2}$ de 2,0 para $4,0 \mathrm{kPa}$. Contudo, no presente trabalho, a elevação nos níveis de $\mathrm{CO}_{2}$ de $<0,5 \mathrm{kPa}$ para $2 \mathrm{kPa}$ pode não ter sido suficiente para causar redução na incidência de podridões em pós-colheita.

A incidência de podridão carpelar foi menor nos frutos armazenados na condição de $1,2 \mathrm{kPa} \mathrm{O}_{2}$ e $2,0 \mathrm{kPa} \mathrm{CO}_{2}$ (Tabela 4), em Vacaria, no segundo ano de avaliação. Não foi constatada podridão carpelar no primeiro ano, em maçãs colhidas nas três regiões e nos dois anos, em frutos do pomar de São Joaquim. Como o início da infecção dos frutos pelos patógenos associados à podridão carpelar ocorre no campo, as condições de armazenamento possuem menor influência sobre a evolução da doença na pós-colheita (Brackmann et al., 2000). No entanto, no presente trabalho obteve-se um bom controle dessa podridão, com $2,0 \mathrm{kPa}$ de $\mathrm{CO}_{2}$, quando essa doença manifestou-se nos frutos provenientes de Vacaria.

A degenerescência de polpa foi maior nos frutos armazenados na condição com $1,2 \mathrm{kPa}$ de $\mathrm{O}_{2}$ e 2,0 kPa de $\mathrm{CO}_{2}$, nos dois anos avaliados (Tabela 4). Esses resultados estão de acordo com os obtidos por Brackmann et al. (1999), que verificaram alta sensibilidade ao $\mathrm{CO}_{2} \mathrm{em}$ maçã 'Fuji' durante o armazenamento em AC. Park \& Lee (2008) também observaram maior incidência de degenerescência da polpa em condições de baixa pressão de $\mathrm{O}_{2}$ e alta de $\mathrm{CO}_{2}$. A redução excessiva de
$\mathrm{O}_{2}$ e aumento do $\mathrm{CO}_{2}$, durante o armazenamento, pode prejudicar a qualidade das maçãs, em grande parte por causa da mudança da respiração aeróbica para a respiração anaeróbica. Essa mudança no metabolismo pode resultar no acúmulo de substâncias voláteis e induzir a manifestação de distúrbios fisiológicos como a degenerescência de polpa (Pedreschi et al., 2009).

A ocorrência de degenerescência de polpa foi maior nos frutos provenientes do pomar de São Joaquim, SC, nos dois anos (Tabela 4). Para Ferguson et al. (1999), há consideráveis variações na incidência de degenerescência da polpa, entre anos de produção, regiões e pomares, independentemente dos efeitos do estádio de maturação dos frutos. Os autores também afirmam que fatores pré-colheita predispõem ao desenvolvimento desse distúrbio, quando os frutos são armazenados em condições de $\mathrm{AC}$ com alta pressão de $\mathrm{CO}_{2}$ e baixa de $\mathrm{O}_{2}$. Apesar de alguns trabalhos relacionarem a incidência de degenerescência da polpa com presença de pingo-de-mel (Brackmann et al., 2002a), no presente trabalho isso não ocorreu. Também não foi verificada correlação significativa entre os atributos de qualidade dos frutos avaliados na colheita e a ocorrência de degenerescência da polpa. Contudo, a incidência da degenerescência da polpa, tanto em baixa $(<0,5 \mathrm{kPa})$ quanto em alta $(2,0 \mathrm{kPa})$ pressão de $\mathrm{CO}_{2}$, correlacionou-se às temperaturas médias que ocorreram durante o desenvolvimentos dos frutos (Tabela 5).

Tabela 4. Ocorrência de podridões e degenerescência de polpa em maçãs 'Fuji' após oito meses de armazenamento em duas condições de atmosfera controlada, e mais sete dias de exposição dos frutos a $20^{\circ} \mathrm{C}^{(1)}$.

\begin{tabular}{|c|c|c|c|c|c|c|c|}
\hline \multirow[t]{2}{*}{ Pressões parciais de $\mathrm{O}_{2}$ e $\mathrm{CO}_{2}$} & \multicolumn{3}{|c|}{$2006 / 2007$} & & \multicolumn{3}{|c|}{$2007 / 2008$} \\
\hline & Vacaria & São Joaquim & Lages & Média & Vacaria & São Joaquim & Média \\
\hline & \multicolumn{7}{|c|}{ Podridões (\%) } \\
\hline \multicolumn{8}{|l|}{ Saída da câmara } \\
\hline $1,2 \mathrm{kPa}$ de $\mathrm{O}_{2}+<0,5 \mathrm{kPa}$ de $\mathrm{CO}_{2}$ & $-(2)$ & - & - & $2,97 \mathrm{a}$ & - & - & $0,70 \mathrm{a}$ \\
\hline $1,2 \mathrm{kPa}$ de $\mathrm{O}_{2}+2,0 \mathrm{kPa}$ de $\mathrm{CO}_{2}$ & - & - & - & $6,01 \mathrm{a}$ & - & - & $2,98 \mathrm{a}$ \\
\hline $\mathrm{CV}(\%)$ & \multicolumn{4}{|c|}{15,2} & \multicolumn{3}{|c|}{47,2} \\
\hline \multicolumn{8}{|l|}{ Após sete dias a $20^{\circ} \mathrm{C}$} \\
\hline $1,2 \mathrm{kPa}$ de $\mathrm{O}_{2}+<0,5 \mathrm{kPa}$ de $\mathrm{CO}_{2}$ & - & - & - & $15,7 \mathrm{a}$ & - & - & $4,10 \mathrm{a}$ \\
\hline $1,2 \mathrm{kPa}$ de $\mathrm{O}_{2}+2,0 \mathrm{kPa}$ de $\mathrm{CO}_{2}$ & - & - & - & $23,8 \mathrm{a}$ & - & - & $2,79 \mathrm{a}$ \\
\hline $\mathrm{CV}(\%)$ & \multicolumn{4}{|c|}{24,5} & \multicolumn{3}{|c|}{69,6} \\
\hline & \multicolumn{7}{|c|}{ Podridão carpelar (\%) } \\
\hline $1,2 \mathrm{kPa}$ de $\mathrm{O}_{2}+<0,5 \mathrm{kPa}$ de $\mathrm{CO}_{2}$ & - & - & - & 0,00 & $13,2 \mathrm{a}$ & 0,00 & $6,6 a$ \\
\hline $1,2 \mathrm{kPa}$ de $\mathrm{O}_{2}+2,0 \mathrm{kPa}$ de $\mathrm{CO}_{2}$ & - & - & - & 0,00 & $3,9 \mathrm{~b}$ & 0,00 & $2,0 \mathrm{~b}$ \\
\hline \multirow[t]{2}{*}{ CV $(\%)$} & & & & & & 45,6 & \\
\hline & \multicolumn{7}{|c|}{ Degenerescência de polpa (\%) } \\
\hline $1,2 \mathrm{kPa}$ de $\mathrm{O}_{2}+<0,5 \mathrm{kPa}$ de $\mathrm{CO}_{2}$ & 1,14 & 4,54 & 1,09 & $2,57 b$ & 0,00 & 8,30 & $4,2 b$ \\
\hline $1,2 \mathrm{kPa}$ de $\mathrm{O}_{2}+2,0 \mathrm{kPa}$ de $\mathrm{CO}_{2}$ & 4,85 & 14,70 & 3,41 & $7,65 \mathrm{a}$ & 14,70 & 30,40 & $22,5 \mathrm{a}$ \\
\hline Média & $2,99 \mathrm{~B}$ & $9,62 \mathrm{~A}$ & $2,25 \mathrm{~B}$ & & $7,35 \mathrm{~B}$ & $19,35 \mathrm{~A}$ & \\
\hline CV (\%) & \multicolumn{4}{|c|}{61,4} & \multicolumn{3}{|c|}{74,8} \\
\hline
\end{tabular}

Pesq. agropec. bras., Brasília, v.45, n.6, p.531-538, jun. 2010 
Tabela 5. Temperaturas médias registradas durante diferentes fases de desenvolvimento dos frutos, nos anos agrícolas de 2006/2007 e 2007/2008, em três regiões de produção, e coeficientes de correlação (r) entre as temperaturas médias e a incidência de degenerescência da polpa em maçãs 'Fuji', colhidas nos diferentes pomares e armazenadas em duas condições de atmosfera controlada.

\begin{tabular}{|c|c|c|c|c|c|c|c|}
\hline \multirow[t]{3}{*}{ Dias após floração ou colheita } & \multicolumn{5}{|c|}{ Temperaturas médias $\left({ }^{\circ} \mathrm{C}\right)$} & \multicolumn{2}{|c|}{$\mathrm{r}$} \\
\hline & \multicolumn{2}{|c|}{$2006 / 2007$} & \multicolumn{3}{|c|}{$2007 / 2008$} & \multirow{2}{*}{$\begin{array}{c}1,2 \mathrm{kPa} \text { de } \mathrm{O}_{2}+<0,5 \mathrm{kPa} \\
\mathrm{de} \mathrm{CO}_{2}\end{array}$} & \multirow{2}{*}{$\begin{array}{c}1,2 \mathrm{kPa} \text { de } \mathrm{O}_{2}+2,0 \mathrm{kPa} \\
\text { de } \mathrm{CO}_{2}\end{array}$} \\
\hline & Lages & Vacaria & São Joaquim & Vacaria & São Joaquim & & \\
\hline 30 dias após plena floração & 17,5 & 18,4 & 17,3 & 18,1 & 17,2 & $-0,76^{\mathrm{ns}}$ & $-0,51^{\mathrm{ns}}$ \\
\hline 60 dias após plena floração & 17,4 & 17,9 & 17,0 & 17,8 & 16,8 & $-0,92 *$ & $-0,68^{\mathrm{ns}}$ \\
\hline 90 dias após plena floração & 19,9 & 18,9 & 18,2 & 18,8 & 17,8 & $-0,74^{\mathrm{ns}}$ & $-0,88^{*}$ \\
\hline 120 dias após plena floração & 20,0 & 19,5 & 18,8 & 19,4 & 18,1 & $-0,85^{\mathrm{ns}}$ & $-0,94 *$ \\
\hline 150 dias após plena floração & 20,4 & 19,6 & 19,0 & 19,5 & 18,3 & $-0,81^{\mathrm{ns}}$ & $-0,92 *$ \\
\hline 180 dias após plena floração & 20,0 & 19,4 & 19,3 & 19,4 & 18,4 & $-0,78^{\mathrm{ns}}$ & $-0,92 *$ \\
\hline 210 dias após plena floração & 19,0 & 19,4 & 19,0 & 18,9 & 18,0 & $-0,71^{\mathrm{ns}}$ & $-0,84^{*}$ \\
\hline 30 dias após colheita & 12,5 & 19,2 & 17,5 & 18,6 & 15,4 & $0,50^{\mathrm{ns}}$ & $-0,07^{\mathrm{ns}}$ \\
\hline 60 dias após colheita & 15,4 & 18,9 & 19,1 & 17,4 & 17,2 & $0,60^{\text {ns }}$ & $-0,05^{\mathrm{ns}}$ \\
\hline
\end{tabular}

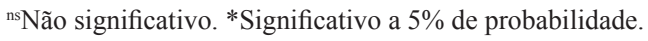

Não houve correlação com a umidade relativa nem com a precipitação nos pomares, em ambos os anos.

A ocorrência de degenerescência da polpa em maçãs 'Fuji', armazenadas sob baixa pressão de $\mathrm{CO}_{2}$ $(<0,5 \mathrm{kPa})$, apresentou correlação negativa com as temperaturas médias obtidas aos 60 dias após a plena floração (DAPF) e não apresentou correlação significativa com as temperaturas nos outros períodos de desenvolvimento dos frutos (Tabela 5). Na condição de $1,2 \mathrm{kPa}$ de $\mathrm{O}_{2}$ e 2,0 $\mathrm{kPa}$ de $\mathrm{CO}_{2}$, a ocorrência de degenerescência da polpa apresentou correlação negativa com as temperaturas médias que ocorreram de 90 a 210 DAPF, e os maiores coeficientes de correlação foram obtidos com as temperaturas médias aos 120, 150 e 180 DAPF. Não houve correlação com as temperaturas máximas e mínimas registradas nos pomares.

Segundo Ferguson et al. (1999), a temperatura durante a fase de maturação dos frutos exerce forte influência sobre o desenvolvimento da degenerescência de polpa. Lau (1997) cita que a degenerescência de polpa, em frutos armazenados em AC, ocorre em razão do adensamento das células, o que prejudica as trocas gasosas. Esse autor também considera que a menor temperatura do ar, durante a fase de desenvolvimento dos frutos, pode provocar esse adensamento das células. Esse fato pode explicar o aumento da suscetibilidade ao dano por $\mathrm{CO}_{2}$, em consequência de temperaturas médias mais baixas durante o desenvolvimento dos frutos, observado no presente trabalho.

\section{Conclusões}

1. A condição de armazenamento de maçãs 'Fuji', em atmosfera controlada com $1,2 \mathrm{kPa}$ de $\mathrm{O}_{2}$ e $2,0 \mathrm{kPa}$ de $\mathrm{CO}_{2}$, mantém os atributos de qualidade dos frutos, não afeta a incidência de podridões e aumenta a ocorrência de degenerescência de polpa.

2. A precipitação pluviométrica e a umidade relativa, durante o desenvolvimento dos frutos no pomar, não têm efeito sobre a suscetibilidade de degenerescência de polpa em maçã 'Fuji'.

3. Temperaturas médias mais baixas durante o desenvolvimento dos frutos aumentam a suscetibilidade de maçãs 'Fuji' à degenerescência de polpa, durante o armazenamento em atmosfera controlada.

\section{Agradecimentos}

Ao Conselho Nacional de Desenvolvimento Científico e Tecnológico e ao Programa de Apoio à Pesquisa da Universidade do Estado de Santa Catarina, pelo apoio financeiro.

\section{Referências}

BLANKENSHIP, S.M.; DOLE, J.M. 1-methylcyclopropene: a review. Postharvest Biology and Technology, v.28, p.1-25, 2003.

BOWEN, J.H.; WATKINS, C.B. Fruit maturity, carbohydrate and mineral content relationships with watercore in Fuji apples. Postharvest Biology and Technology, v.11, p.31-38, 1997.

BRACKMANN, A.; BENEDETTI, M.; STEFFENS, C.A.; MELLO, A.M. de. Efeito da temperatura e condições de atmosfera controlada na armazenagem de maçãs Fuji com incidência de 
pingo-de-mel. Revista Brasileira de Agrociência, v.8, p.37-42, 2002a.

BRACKMANN, A.; BORTOLUZZI, G.; BORTOLUZ, L. Controle da degenerescência da polpa da maçã Fuji com concentrações dinâmicas de $\mathrm{O}_{2}$ e $\mathrm{CO}_{2}$ e redução da umidade relativa durante o armazenamento em atmosfera controlada. Ciência Rural, v.29, p.459-463, 1999.

BRACKMANN, A.; GIEHL, R.F.H.; ANTES, R.B.; NEUWALD, D.A.; SESTARI, I.; PINTO, J.A.V. Condições de atmosfera controlada para o armazenamento de maçãs Royal Gala de diferentes tamanhos. Ciência Rural, v.35, p.1049-1053, 2005a.

BRACKMANN, A.; GIEHL, R.F.H.; SESTARI, I.; STEFFENS, C.A. Condições de atmosfera controlada, temperatura e umidade relativa no armazenamento de maçãs Fuji. Ciência e Agrotecnologia, v.29, p.803-809, 2005b.

BRACKMANN, A.; GUARIENTI, A.J.W.; SAQUET, A.A.; GIEHL, R.F.H.; SESTARI, I. Condições de atmosfera controlada para a maçã Pink Lady. Ciência Rural, v.35, p.504-509, 2005c.

BRACKMANN, A.; STEFFENS, C.A.; WACLAWOSKY, A.J. Efeito da temperatura e da pressão parcial de $\mathrm{CO}_{2}$ sobre maçã Gala armazenada sob atmosfera controlada contendo pressão parcial ultra-baixa de oxigênio. Ciência e Agrotecnologia, v.24, p.25-32, 2000 .

BRACKMANN, A.; STEFFENS, C.A.; WACLAWOSKY, A.J. Influência da época de colheita e do armazenamento em atmosfera controlada na qualidade da maçã Braeburn. Pesquisa Agropecuária Brasileira, v.37, p.295-301, 2002b.

CLARCK, C.J.; MCGLONE, V.A.; JORDAN, R.B. Detection of brownheart in Braeburn apple by transmission NIR spectroscopy. Postharvest Biology and Technology, v.28, p.87-96, 2003.

FERGUSON, I.; VOLZ, R.; WOOLF, A. Preharvest factors affecting physiological disorders of fruit. Postharvest Biology and Technology, v.15, p.255-262, 1999.

KONOPACKA, D.; PLOCHARSKI, W.J. Effect of storage conditions on the relationship between apple firmness and texture acceptability. Postharvest Biology and Technology, v.32, p.205-211, 2004.
KRETZSCHMAR, A.A.; MARODIN, G.A.B.; DUARTE, V.; VALDEBENITO SANHUEZA, R.M.; GUERRA, D.S. Efeito de fitorreguladores sobre a incidência de podridão carpelar em maçãs Fuji. Revista Brasileira de Fruticultura, v.29, p.414-419, 2007.

LAU, O.L. Influence of climate, harvestmaturity, waxing, $\mathrm{O}_{2}$ and $\mathrm{CO}_{2}$ on browning disorders of Braeburn apples. In: INTERNATIONAL CONTROLLED ATMOSPHERE RESEARCH CONFERENCE, 7., 1997, Davis. Proceedings. Davis: University of California, 1997. v.2, p.132-137.

PARK, Y.-M.; LEE, Y.-J. Induction of modified atmosphere-related browning disorders in Fuyu persimmon fruit. Postharvest Biology and Technology, v.47, p.346-352, 2008.

PEDRESCHI, R.; FRANCK, C.; LAMMERTYN, J.; ERBAN, A.; KOPKA, J.; HERTOG, M.; VERLINDEN, B.; NICOLAI, B. Metabolic profiling of Conference pears under low oxygen stress. Postharvest Biology and Technology, v.51, p.123-130, 2009.

PEDRESCHI, R.; HERTOG, M.; ROBBEN, J.; NOBEN, J.-P.; NICOLAI, B. Physiological implications of controlled atmosphere storage of Conference pears (Pyrus communis L.): a proteomic approach. Postharvest Biology and Technology, v.50, p.110-116, 2008.

SANHUEZA, R.M.V.; BETTI, J.A. Doenças da macieira. In: KIMATI, H.; AMORIM, L.; REZENDE, J.A.M.; BERGAMIN FILHO, A.; CAMARGO, L.E.A. (Org.). Manual de fitopatologia: doenças das plantas cultivadas. 4.ed. São Paulo: Agronômica Ceres, 2005. p.421-433.

SAQUET, A.; STREIF, J. Respiração e produção de etileno de maçãs armazenadas em diversas concentrações de oxigênio. Revista Brasileira de Agrociência, v.8, p.71-75, 2002.

STEFFENS, C.A.; BRACKMANN, A.; PINTO, J.A.V.; EISERMANN, A.C. Taxa respiratória de frutas de clima temperado. Pesquisa Agropecuária Brasileira, v.42, p.313-321, 2007.

STEFFENS, C.A.; GUARIENTI, A.J.W.; STORCK, L.; BRACKMANN, A. Maturação da maçã 'Gala' com a aplicação pré-colheita de aminoetoxivinilglicina e ethephon. Ciência Rural, v.36, p.434-440, 2006.

STREIF, J. Jod-Stärke-Test zur Beurteilung der Fruchtreife bei Äpfeln. Obst und Garten, v.8, p.382-384, 1984.

Recebido em 15 de março de 2010 e aprovado em 10 de maio de 2010

Pesq. agropec. bras., Brasília, v.45, n.6, p.531-538, jun. 2010 